\title{
Energy shifts of spectral lines from accretion discs near a black hole with negative spin
}

\section{Vjačeslav Sochora}

Astronomical Institute, Academy of Sciences, Prague

E-mail: sochoraeastro.cas.cz

\section{Vladimír Karas}

Astronomical Institute, Academy of Sciences, Prague

E-mail: vladimir.karas@cuni.cz

Spectral lines are a powerful tool to gain important information about black holes in X-ray binaries and active galactic nuclei. We present a semi-analytical solution to determine the observed energy range of such relativistic spectral lines as a function of the model parameters, namely, the emission radius, observer's view angle, and the black hole spin. This is a continued work, where we already presented the solution for the systems with black holes with positive spin. Now we study the case of systems with negative spin. We compare the results for these two different cases and discuss the dependence of minimum and maximum energy shifts on the model parameters.

25th Texas Symposium on Relativistic Astrophysics - TEXAS 2010

December 06-10, 2010

Heidelberg, Germany 


\section{Introduction}

The observation of spectral lines from inner regions of accretion disks around black hole gives us information about this matter in extreme conditions. $\mathrm{K} \alpha$ spectral line of iron, broadened and skewed by fast orbital motion and redshifted by strong gravitational field, has been used to constrain parameters of the black hole, both in active galactic nuclei [10], [19], [16] and Galactic X-ray binaries containing black-holes [15], [14]. Most of these cases suppose prograde rotation of the black hole. In our theoretical work we present the results for the case of the black hole with the negative spin [8] and compare it with the case of the positive spin [12].

We can imagine the accretion disk as a superposition of radiating accretion rings that extend from the inner edge to the outer rim of the disk, with a prescribed profile of emissivity as a function of radius. On one hand such a superposition can be seen as a merely formal way of integrating the total signal from the accretion disk that extends over the range of radii. The convenience of this formulation arises naturally from the fact that we usually consider perfectly circular motion of the emitting material, which only very slowly spirals toward the centre (eccentric rings are only rarely discussed in the literature). Therefore, the emission originates effectively from a set of infinitesimally narrow, concentric rings which sum up to form the total signal.

On the other hand, a relatively narrow range of emission radii may indeed represent a realistic emission profile under certain circumstances. This is for example the case of so called flare/spot scenario [7], [9], [11]. According to this scheme, magnetically triggered flares occur above the disk surface due to reconnection of field lines twisted by differential rotation. Illumination of the disk then produces the reflection spectral line that emerges from a spot at a well defined radius in the disk. As the flare orbits with the underlying disk it creates a ring of emission, azimuthal extent of which is determined by the interplay between duration of the observation and the orbital period at the corresponding radius.

The exposure time is governed by technical specification of the detector and brightness of the source. As for the orbital period, Keplerian motion (which we assume) near a rotating black hole is determined by distance from the centre and the spin of the black hole. The latter causes asymmetry between the cases of prograde versus retrograde motion (a purely general relativistic effect). Although prograde rotation (i.e. a positive spin) is often assumed, counter-rotation (negative spin) cannot be excluded a priori. In fact, the orientation of the black hole rotation with respect to the accretion disk is mainly defined by the boundary condition at the outer edge of the system. Therefore, it makes sense to include both positive and negative spin into consideration.

There is currently a lot of uncertainty concerning the maximum life-time of the flares illumination the disk below. Because of significant shearing of the magnetic loops, it has been assumed that flares can last typically a fraction of the orbital period at the corresponding radius, but exceptionally prominent and persistent flares seem to occur occasionally in the observed lightcurves [18]. Another relevant point in this respect is that actually the individual flares do not have to last for the entire period, instead a sequence of causally triggered events can develop from the parent flare [17]. These "offsprings" should all occur at a similar radius like the original parent flare, producing the reflection line at approximately the same distance from the black hole.

In our work we show straightforward method to calculate extremal energy shifts of radiation from accretion rings lying in equatorial plane. The calculated extremal energy shifts are presented 
in contour graphs for different observer's view angle.

\section{Photon propagation in Kerr metric}

The gravitational field of a stationary, axisymetric and rotating black hole is described by Kerr metric [5], [13]. The form of Kerr metric in Boyer-Lindquist co-ordinates $(t, r, \theta, \phi)$ and geometrised units $(c=G=M=1)$ is

$$
d s^{2}=-\left(1-\frac{2 r}{\Sigma}\right) \mathrm{d} t^{2}-\frac{4 a r}{\Sigma} \sin ^{2} \theta \mathrm{d} t \mathrm{~d} \phi+\frac{A}{\Sigma} \sin ^{2} \theta \mathrm{d} \phi^{2}+\frac{\Sigma}{\Delta} \mathrm{d} r^{2}+\Sigma \mathrm{d} \theta^{2},
$$

where

$$
\Sigma=r^{2}+a^{2} \cos ^{2} \theta ; \Delta=r^{2}-2 r+a^{2} ; A=\left(r^{2}+a^{2}\right)^{2}-a^{2} \Delta \sin ^{2} \theta .
$$

The metric depends only on one parameter, angular momentum of the black hole $a$.

The minimum allowed radius of a stable circular equatorial orbit, so called marginally stable orbit [2], is given by the roots of the equation

$$
r^{2}-6 r \mp 8 a \sqrt{r}-3 a^{2}=0 .
$$

The roots are

$$
r_{\mathrm{ms}}=3+Z_{2} \mp\left[\left(3-Z_{1}\right)\left(3+Z_{1}+2 Z_{2}\right)\right]^{1 / 2}
$$

where $Z_{1}=1+\left(1-a^{2}\right)^{1 / 3}\left[(1+a)^{1 / 3}+(1-a)^{1 / 3}\right] ; Z_{2}=\left(3 a^{2}+Z_{1}^{2}\right)^{1 / 2}$, where the upper sign refers to co-rotating and the lower to counter-rotating orbits.

The path of photons (null geodesic) in Kerr metric is completely described by three constants of motion: the total energy $E$, the azimuthal angular momentum $L_{\mathrm{z}}$, and Carter's constant $Q$. We can further reduce the number of constants by re-normalizing $L_{z}$ and $Q$ with respect to energy $E$, $\lambda=\frac{L_{z}}{E}, q^{2}=\frac{Q}{E^{2}}$.

Further, a null geodesic must satisfy the Carter equation [4]

$$
\pm \int_{r} \frac{\mathrm{d} r}{\sqrt{R\left(r, \lambda, q^{2}\right)}}= \pm \int_{\mu} \frac{\mathrm{d} \mu}{\sqrt{\Theta\left(\mu, \lambda, q^{2}\right)}}
$$

where

$$
R(r)=r^{4}+\left(a^{2}-\lambda^{2}-q^{2}\right) r^{2}+2\left[q^{2}+(\lambda-a)^{2}\right] r-a^{2} q^{2}
$$

and

$$
\Theta\left(\mu, \lambda, q^{2}\right)=q^{2}+\left(a^{2}-\lambda^{2}-q^{2}\right) \mu^{2}-a^{2} \mu^{4},
$$

where we suppose the substitution $\mu=\cos \theta$. The left side of the eq. (2.5) describes the motion in radial direction and the right side the motion in latitudinal direction.

The roots of $R(r)$ and $\Theta(\mu)$ correspond to the turning points in radial and latitudinal directions respectively, see [6] and [12].

The integrals in Carter equation (2.5) can be expressed in the form of the elliptical integrals of first kind [1], [3]

$$
F(\varphi, k)=\int_{0}^{\varphi} \frac{\mathrm{d} \vartheta}{\sqrt{1-k \sin ^{2} \vartheta}} .
$$

The explicit form of the integral depends on whether the roots are real or complex and whether the photon passes through the turning points or not. 


\subsection{The radial integral, real roots}

The case of real roots is

$$
\int_{r_{\mathrm{e}}}^{\infty} \frac{\mathrm{d} r}{\sqrt{R\left(r, \lambda, q^{2}\right)}}=g_{r}\left[F\left(\varphi_{\mathrm{o}}, k_{r}\right) \pm F\left(\varphi_{\mathrm{e}}, k_{r}\right)\right]
$$

where

$$
\begin{gathered}
g_{r}\left(\lambda, q^{2}\right)=\frac{2}{\sqrt{\left(r_{1}-r_{3}\right)\left(r_{2}-r_{4}\right)}} ; k_{r}\left(\lambda, q^{2}\right)=\frac{\left(r_{2}-r_{3}\right)\left(r_{1}-r_{4}\right)}{\left(r_{1}-r_{3}\right)\left(r_{2}-r_{4}\right)}, \\
\varphi_{\mathrm{o}}\left(\lambda, q^{2}\right)=\arcsin \left(\sqrt{\frac{r_{2}-r_{4}}{r_{1}-r_{4}}}\right) ; \varphi_{\mathrm{e}}\left(\lambda, q^{2}\right)=\arcsin \left[\sqrt{\frac{\left(r_{2}-r_{4}\right)\left(r_{\mathrm{e}}-r_{1}\right)}{\left(r_{1}-r_{4}\right)\left(r_{\mathrm{e}}-r_{2}\right)}}\right],
\end{gathered}
$$

$r_{1}, r_{2}, r_{3}, r_{4}$ are the roots of $R(r)$ and $r_{\mathrm{e}}$ is the radius of an accretion ring, see [12]. The upper sign refers to the case of a photon that passes through the turning point and the lower sing refers to the case of a photon that does not pass through the turning point in the radial direction.

\subsection{The radial integral, complex roots}

We suppose $r_{1}, r_{2}$ are complex and $r_{3}, r_{4}$ are real, then the roots $r_{1}, r_{2}$ can be written in the form $r_{1}=u+i v, r_{2}=u-i v$. The expression for this case is

$$
\int_{r_{\mathrm{e}}}^{\infty} \frac{\mathrm{d} r}{\sqrt{R\left(r, \lambda, q^{2}\right)}}=g_{r}\left[F\left(\varphi_{\mathrm{o}}, k_{r}\right)-F\left(\varphi_{\mathrm{e}}, k_{r}\right)\right]
$$

where

$$
\begin{gathered}
g_{r}\left(\lambda, q^{2}\right)=\frac{1}{\sqrt{A B}} ; k_{r}\left(\lambda, q^{2}\right)=\frac{(A+B)^{2}-\left(r_{3}-r_{4}\right)^{2}}{4 A B}, \\
\varphi_{\mathrm{o}}\left(\lambda, q^{2}\right)=\arccos \left[\frac{A-B}{A+B}\right] ; \varphi_{\mathrm{e}}\left(\lambda, q^{2}\right)=\arccos \left[\frac{(A-B) r_{\mathrm{e}}+r_{3} B-r_{4} A}{(A+B) r_{\mathrm{e}}-r_{3} B-r_{4} A}\right], \\
A\left(\lambda, q^{2}\right)=\left[\left(r_{3}-u\right)^{2}+v^{2}\right]^{1 / 2} ; B\left(\lambda, q^{2}\right)=\left[\left(r_{4}-u\right)^{2}+v^{2}\right]^{1 / 2} .
\end{gathered}
$$

\subsection{The latitudinal integral}

The form of the latitudinal integral is

$$
\int_{0}^{\mu_{0}} \frac{\mathrm{d} \mu}{\sqrt{\Theta\left(\mu, \lambda, q^{2}\right)}}=\frac{g_{\mu}}{|a|} F\left(\psi, k_{\mu}\right)
$$

if the photon does not pass through the turning point. For the case with transit through the turning point it is

$$
\int_{0}^{\mu_{0}} \frac{\mathrm{d} \mu}{\sqrt{\Theta\left(\mu, \lambda, q^{2}\right)}}=\frac{g_{\mu}}{|a|}\left[2 K\left(k_{\mu}\right)-F\left(\psi, k_{\mu}\right)\right]
$$

where

$$
g_{\mu}\left(\lambda, q^{2}\right)=\frac{1}{\sqrt{\mu_{+}^{2}+\mu_{-}^{2}}} ; k_{\mu}\left(\lambda, q^{2}\right)=\frac{\mu_{+}^{2}}{\mu_{+}^{2}+\mu_{-}^{2}} ; \psi\left(\lambda, q^{2}\right)=\arcsin \left[\sqrt{\frac{\mu_{\mathrm{o}}^{2}\left(\mu_{+}^{2}+\mu_{-}^{2}\right)}{\mu_{+}^{2}\left(\mu_{\mathrm{o}}^{2}+\mu_{-}^{2}\right)}}\right],
$$

$\mu_{+}^{2}, \mu_{-}^{2}$ are the roots of $\Theta(\mu), \mu_{\mathrm{o}}$ is observer's view angle and $K\left(k_{\mu}\right)=F\left(\frac{\pi}{2}, k_{\mu}\right)$. 


\section{Extremal photon energy shifts}

The energy shift is defined as the ratio

$$
g(\lambda)=\frac{E_{\mathrm{o}}}{E_{\mathrm{e}}}=\frac{1}{u^{t}} \frac{1}{1-\lambda \Omega}
$$

between the observed $E_{\mathrm{o}}$ and emitting $E_{\mathrm{e}}$ energy. The emission source orbits the black hole at Keplerian four-velocity $\mathbf{u}=u^{t}(1,0,0, \Omega)$, where $u^{t}=\left[1-2 r_{\mathrm{e}}^{-1}(1-a \Omega)^{2}-\left(r_{\mathrm{e}}^{2}+a^{2}\right) \Omega^{2}\right]^{-1 / 2}$ and $\Omega\left(r_{e}\right)=\left(r_{e}^{3 / 2}+a\right)^{-1}$ is the Keplerian angular velocity at the emission radius $r_{e}$ and $a$ is the spin of black hole.

The extreme values of $g$ are required to meet the conditions of the Carter equation (2.5). We used the Lagrange multipliers $\alpha$ to find their values. Define the Lagrangian as

$$
\Lambda\left(\lambda, q^{2}, \alpha\right)=\frac{1}{u^{t}} \frac{1}{1-\lambda \Omega}-\alpha \int_{r_{\mathrm{e}}}^{\infty} \frac{\mathrm{d} r}{\sqrt{R\left(r, \lambda, q^{2}\right)}}+\alpha \int_{0}^{\mu_{\mathrm{o}}} \frac{\mathrm{d} \mu}{\sqrt{\Theta\left(\mu, \lambda, q^{2}\right)}} .
$$

The partial derivatives of the Lagrange function (3.2) with respect to $\lambda, q^{2}$ and $\alpha$ must vanish identically. The latter condition yields two equations for two unknowns $\left(\lambda, q^{2}\right)$,

$$
\begin{gathered}
f_{1}=\int_{r_{\mathrm{e}}}^{\infty} \frac{\mathrm{d} r}{\sqrt{R\left(r, \lambda, q^{2}\right)}}-\int_{0}^{\mu_{\mathrm{o}}} \frac{\mathrm{d} \mu}{\sqrt{\Theta\left(\mu, \lambda, q^{2}\right)}}=0, \\
f_{2}=\frac{\partial f_{1}}{\partial q^{2}}=\frac{\partial}{\partial q^{2}}\left[\int_{r_{\mathrm{e}}}^{\infty} \frac{\mathrm{d} r}{\sqrt{R\left(r, \lambda, q^{2}\right)}}-\int_{0}^{\mu_{0}} \frac{\mathrm{d} \mu}{\sqrt{\Theta\left(\mu, \lambda, q^{2}\right)}}\right]=0 .
\end{gathered}
$$

To solve eqs. (3.3) and (3.4) we used numerical Newton-Raphson method in the form of Taylor expansion about the root. The explicit solution can be found by successive iterations according to the prescription $\lambda_{\mathrm{n}+1}=\lambda_{\mathrm{n}}+\Delta \lambda_{\mathrm{n}}, q_{\mathrm{n}+1}^{2}=q_{\mathrm{n}}^{2}+\Delta q_{n}^{2}$, where

$$
\Delta q_{\mathrm{n}}^{2}=\frac{f_{1} \frac{\partial f_{2}}{\partial \lambda}-f_{2} \frac{\partial f_{1}}{\partial \lambda}}{\frac{\partial f_{1}}{\partial \lambda} \frac{\partial f_{2}}{\partial q^{2}}-\frac{\partial f_{1}}{\partial q^{2}} \frac{\partial f_{2}}{\partial \lambda}}, \Delta \lambda_{\mathrm{n}}=\frac{-f_{1}-\Delta q_{\mathrm{n}}^{2} \frac{\partial f_{1}}{\partial q^{2}}}{\frac{\partial f_{1}}{\partial \lambda}} .
$$

There are always two solutions of pairs $\left(\lambda, q^{2}\right)$, where one corresponds to the minimal energy shift and the second to the maximal energy shift. The extremal energy shifts for different observer's view angles are shown in Fig. 1. The success of this numerical method depends on the right starting value and on the knowledge of the derivatives of the elliptic integrals.

\section{Discusion and Conclusion}

The solution for the case of the negative spin of black hole connects to the solution of the positive spin (Fig. 1). We see that the value of $g_{\max }$ is always bigger for smaller spins than for greater spins and the value of $g_{\min }$ is smaller for smaller spins than for greater spins if we suppose the same radius. That means the width is broader for negative spins than for positive spins. 

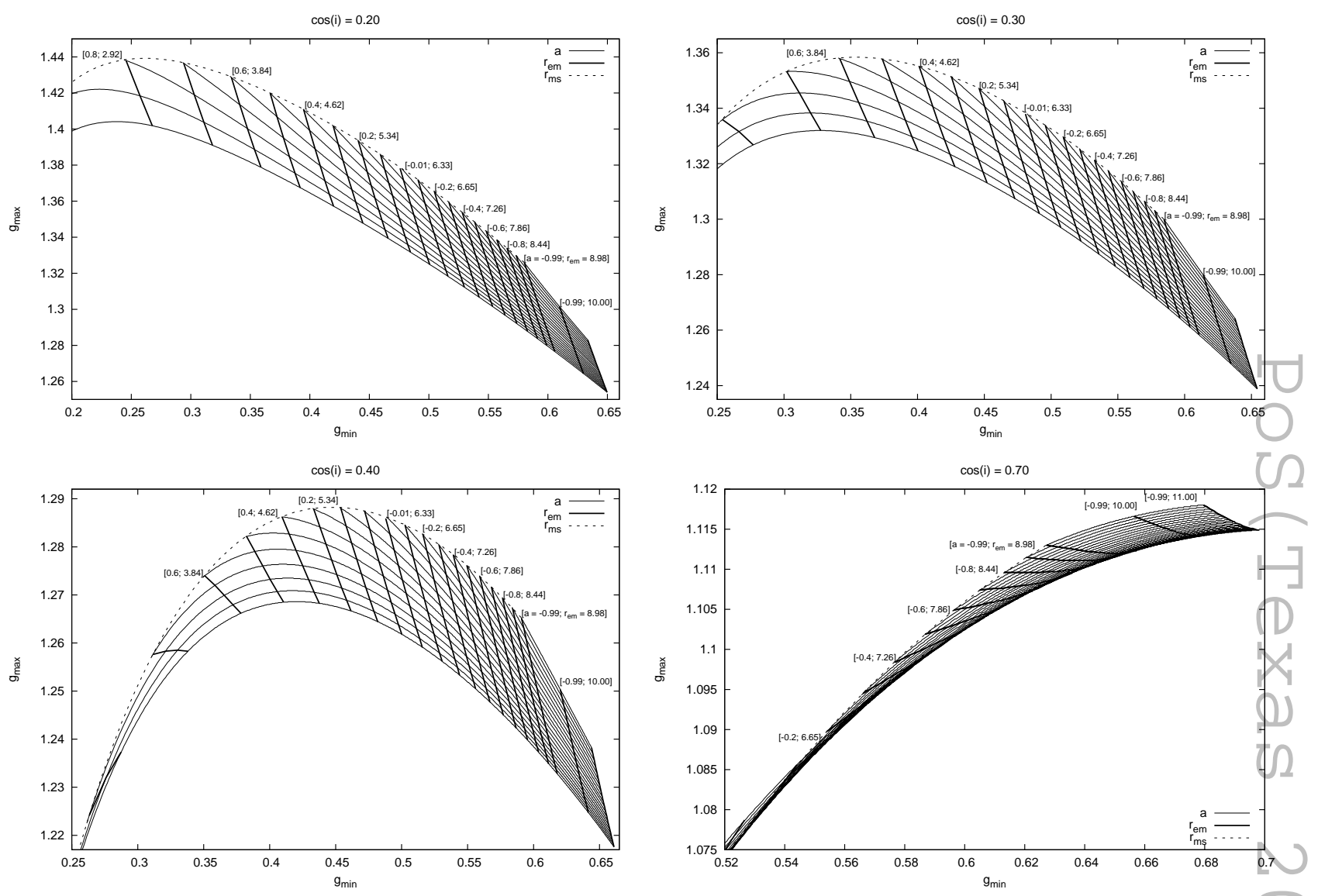

Figure 1: Examples of contour graphs with extremal energy shifts for different view angle of the observer (cosi on the top of graph) and for the negative spin of black hole $a$. Thick lines represent emission radius $r_{\mathrm{em}}$, thin lines the spin of black hole $a$.

The dependence of energy shift on emitting radius is that minimum and maximum of $g$ go always to the value 1 for farther radii. The maximum value of energy shift grows at first with larger emitting radius and then declines to the value 1 . This effect occurs with a small inclination angle.

The maximum values of the energy shift drop and minimum values grow with smaller inclination angle. It is because for smaller view angle there is smaller Doppler redshift and blueshift.

In our work we presented semi-analytic solution to find extremal values of energy shifts of radiation from accretion ring around Kerr's black hole. The solution is written in an implicit form, eqs. (3.3), (3.4) and we used Newton-Raphson numerical method to solve them. It is an iteration procedure with prescription (3.5). The advantages of this method are the quickness and accuracy, but we need to know the derivatives of the elliptical integrals.

\section{Acknowledgments}

The authors acknowledge support from the doctoral grant of the Czech Science Foundation no. 205/09/H033. 


\section{References}

[1] M. Abramowitz, I. A. Stegun, Handbook of Mathematical Functions, Dover, New York 1965.

[2] J. M. Bardeen, W. H. Press, S. A. Teukolsky, Rotating Black Holes: Locally Nonrotating Frames, Energy Extraction, and Scalar Synchrotron Radiation, ApJ 178 (1972), 347-370.

[3] P. F. Byrd, M. D. Friedman, Handbook of Elliptic Integrals for Engineers and Scientists, Springer-Verlag, New York 1971.

[4] B. Carter, Global Structure of the Kerr Family of Gravitational Fields, Phys. Rev. 174 (1968) 1559.

[5] S. Chandrasekhar, The Mathematical Theory of Black Holes, Oxford University Press, Oxford 2004.

[6] A. Čadež, C. Fanton, M. Calvani, Line Emission from Accretion Discs around Black Holes: the Analytic Approach, New Astronomy 3 (1998) 647-654.

[7] B. Czerny, A. Różańska, M. Dovčiak, V. Karas, A. M. Dumont, The Structure and Radiation Spectra of Illuminated Accretion Disks in AGN. II. Flare/spot Model of X-ray Variability, A\&A 420 (2004) 16.

[8] T. Dauser, J. Wilms, C. S. Reynolds, L. W. Brenneman, Broad Emission Lines for a Negatively Spinning Black Hole, MNRAS 409 (2010) 1534-1540.

[9] M. Dovčiak, V. Karas, G. Matt, R. W. Goosmann, Variation in the Primary and Reprocessed Radiation from an Orbiting Spot around a Black Hole, MNRAS 384 (2008) 361.

[10] A. C. Fabian, K. Iwasawa, C. S. Reynolds, A. J. Young, Broad Iron Lines in Active Galactic Nuclei, PASP 112 (2000) 1145-1161.

[11] P. Jovanović, L. Č. Popović, M. Stalevski, A. I. Shapovalova, Variability of the H $\beta$ Line Profiles as an Indicator of Orbiting Bright Spots in Accretion Disks of Quasars: A Case Study of 3C 390.3, ApJ 718 (2010) 168.

[12] V. Karas, V. Sochora, Extremal Energy Shifts of Radiation from a Ring Near a Rotating Black Hole, ApJ 725 (2010) 1507-1515.

[13] R. P. Kerr, Gravitational Field of a Spinning Mass as an Example of Algebraically Special Metric, Phys. Rev. Lett. 11 (1963) 237.

[14] J. E. McClintock, R. A. Remillard, Compact Stellar X-ray Sources, Cambridge University Press, Cambridge 2006, pp. 157-213.

[15] J. M. Miller, A. C. Fabian, R. Wijnands, R. A. Remillard, P. Wojdowski et al., Resolving the Composite Fe Ka Emission Line in the Galactic Black Hole Cygnus X-1 with Chandra, ApJ, 578 (2002) 348-356.

[16] J. Miller, Relativistic X-Ray Lines from the Inner Accretion Disks Around Black Holes, ARA\&A 45 (2007) 441-479.

[17] T. Pecháček, V. Karas, B. Czerny, Hot-spot Model for Accretion Disc Variability as Random Process, A\&A 487 (2008) 815.

[18] G. Ponti, M. Cappi, M. Dadina, G. Malaguti, Mapping the inner regions of MCG-6-30-15 with XMM-Newton, A\&A, 417 (2004) 451.

[19] C. S. Reynolds, M. A. Nowak, Fluorescent iron lines as a probe of astrophysical black hole systems, Phys. Rep. 377 (2003) 389-466. 